\title{
Secondary traumatic stress and vicarious posttraumatic growth in healthcare workers during the first COVID-19 lockdown in Greece: The role of resilience and coping strategies
}

\author{
Argyroula Kalaitzaki, Michael Rovithis
}

Laboratory of Interdisciplinary Approaches to the Enhancement of Quality of Life, Social Work Department, Hellenic Mediterranean University, Heraklion, Crete, Greece

ARTICLE HISTORY: Received 2 October 2020/Revised 20 December 2020/Published Online 17 March 2021

\begin{abstract}
Despite the indisputable negative psychosocial consequences of the COVID-19 pandemic, positive consequences are also possible. Resilience and coping strategies have been assumed to contribute to these outcomes. However, findings are still scarce and inconclusive. The study aimed to examine the role of resilience and coping strategies in the secondary stress for the Greek healthcare workers (HCWs) and in the posttraumatic growth following the COVID-19 lockdown in Greece. A sample of 673 HCWs coming from Greece were recruited. A convenience and snowball mixed sampling procedure were used. A questionnaire was distributed through social networking sites, webpages, and personal contacts of the author. Participants were asked to distribute it to their own contacts. Recruitment occurred during April 5-30, 2000, amid the lockdown (March 23-May 03), when people were asked to follow the stringent lockdown constraint enforced by the Greek government. Sociodemographic data were collected. The Secondary Traumatic Stress Scale measured secondary traumatic stress (STS) for the HCWs. The PostTraumatic Growth Inventory, the Brief Resilience Scale, and the Coping Orientation to Problems Experienced Inventory measured posttraumatic growth, resilience, and coping strategies, respectively. Regression analyses demonstrated that resilience and coping strategies were differentially associated with positive and negative (stress/growth) lockdown outcomes. Resilience and mostly maladaptive coping strategies predicted STS. A mixture of adaptive and maladaptive coping strategies predicted PTG. The so-called "second wave" of the outbreak that started in August 2020 indicates that the study of the psychosocial impact of the COVID-19 pandemic and lockdown and of the internal resources (resilience and coping) to deal with, is necessary. The findings contribute to a more comprehensive understanding of the coping strategies used by population subgroups (e.g., HCWs) in dealing with the COVID-19 lockdown in Greece. Enhancing internal resources through supportive services will ameliorate HCWs ability to withstand, recover, and thrive with benefits in their psychological health and well-being.
\end{abstract}

KEYWORDS: Coronavirus, vicarious traumatization, vicarious posttraumatic growth, coping responses to stress, Greece.

\section{Introduction}

COVID-19 unexpectedly and rapidly resulted in extremely high rates of infected people worldwide and therefore, soon after its first onset (December 2019) WHO declared COVID-19 a pandemic on March 11, 2020. Governments worldwide imposed measures to limit its spread ranging from social distance to severe lockdown. Greece early enough enforced severe social constraints to prevent the spread of the virus (e.g., cancellation of all cultural/artistic events and cessation of schools), which eventually resulted in lockdown and extreme social distancing ("Stay Home" 
measure) on March 23, 2020, with gradual de-escalation on May 4, 2020.'

Facing a life-threatening disease may have both negative and positive effects. Vicarious Traumatization (or Secondary Traumatic Stress; STS) refers to the symptoms of intrusion, avoidance, and arousal resulted from one's [e.g., healthcare workers' $\left.\left(\mathrm{HCWs}^{\prime}\right)\right]$, indirect exposure to traumatic events experienced by traumatized patients. ${ }^{2}$ Similarly, the positive response or the psychological benefits that HCWs may have from the indirect exposure is called Vicarious Post Traumatic Growth (VPTG). ${ }^{3,4}$ Being constantly exposed to the COVID-19 and its consequences, HCWs are a high-risk group. ${ }^{4}$ Although studies have mostly emphasized the adverse mental and emotional outcomes of the COVID-19 pandemic among HCWs such as anxiety, depression, stress, post-traumatic stress, insomnia, psychological distress, and burnout (see recent meta-analysis), ${ }^{5}$ there is a shortage of research to date on STS, which may profoundly affect wellbeing and quality of life.

Even less is known about VPTG. VPTG is an indication that a traumatic experience does not necessarily result solely in negative consequences, since there are people that cope well. ${ }^{6}$ Mancini ${ }^{7}$ has argued that the pandemic may have some social and mental health benefits for certain groups of people as acute stress may trigger positive responses. Although, a number of authors ${ }^{8,9}$ have suggested the significance of studying PTG among HCWs, only scarce attempts can be found. ${ }^{10,11}$ Thus, any positive outcomes of the COVID-19 pandemic remain to be studied.

Within the limited literature on the resources that may help individuals combat the detrimental consequences of the COVID-19 outbreak, coping strategies and psychological resilience are undoubtedly among the most studied ones. ${ }^{12}$ Problem-focused coping strategies have been considered the most effective responses. ${ }^{13,14}$ Seeking social support, avoidance, and positive appraisal of the situation have also been reported..$^{13}$ Higher resilience and use of humor have been associated with less anxiety, while mental disengagement with higher anxiety in Israelian nursing students. ${ }^{15}$ It has also been found that resilience, adaptive coping strategies, and social support mediates the relationship between COVID-19 stressful experience and acute stress disorder in Chinese students. ${ }^{12}$ However, findings are still scarce and inconclusive. Besides, to the author knowledge, none study as yet has examined the relationship of the coping strategies and resilience with VPTG during the COVID-19 outbreak.

The aim of this study was to examine the association of the personal resources, such as resilience and coping responses, with the positive and negative (VPTG and STS) mental health impact of the COVID-19 lockdown in HCWs in Greece. We hypothesized that resilience and adaptive coping strategies will increase the likelihood of a positive outcome (VPTG), whereas maladaptive coping strategies will be associated with a greater risk of a negative outcome (STS).

By the time of writing this paper and to authors' knowledge, the association of the coping strategies and resilience with the positive and negative psychological outcomes of the COVID-19 lockdown in the HCWs has not yet been reported by any other study in Greece. Hopefully, the study findings will contribute to the accumulating evidence on the use of personal resources to combat the current pandemic, failure of which -in the long run- may have a catastrophic impact on mental health, wellbeing, and quality of life. Since the pandemic's long-term impact may be unforeseen, safeguarding the HCWs who are at increased risk with constructive coping responses to deal with the crisis is of paramount importance.

\section{Material and Method}

\section{Sample}

After excluding two persons (not residing in Greece), the final sample included $673 \mathrm{HCWs}$, coming from all nine geographical regions of Greece. HCWs were all employed $(100 \%)$ in the healthcare sector, mostly females (74.7\%), well-educated (tertiary education 56.9\%), and residing in Crete (47.9\%). They were 43.4 years old $(S D=9.8)$ and mostly with a partner $(62.3 \%)$. They were recruited from the primary (e.g., community health centers, Help at Home) and secondary healthcare system (i.e., hospitals) and were physicians and nurses (77.1\%) or social workers and psychologists (22.9\%). A number of demographic variables correlated with the outcome variables (table 1). Given that this goes beyond the aims of this study, detailed correlations of the demographics with the outcome measures can be found elsewhere. ${ }^{16}$

\section{Procedure}

The data were collected during April 5-30, 2000, amid the lockdown in Greece (March 23-May 03). A convenience and snowball mixed sampling procedure were used. The link of the google forms questionnaire was posted in social networking sites (e.g., Facebook, Linkedln) and webpages, and participants were asked to distribute it to their contacts. The informed consent notified about the aim of the study and participants' rights. The study abides with the ethical principles of the Helsinki Declaration of 1964 and it was approved by the University Ethics Committee, in which the author is affiliated. 


\section{Measures}

Demographic data was collected, and a number of questions asked participants' experience with the traumatic event of COVID-19. HCWs were asked about their level of contact with infected and/or diagnosed patients at work (Yes. No, Likely, Don't know), and responses were coded as 'exposure' (Yes/Likely) or 'no exposure'.

The following measures were also administered: Outcome Measures. The 17-item Secondary Traumatic Stress Scale $(\mathrm{STS})^{2}$ was administered to measure secondary traumatic stress (Cronbach's $a=0.914)$. Items are scored from 1 (Never) to 5 (Very Often). A cumulative score of 3 or greater is considered to be positive for at least a moderate level of STS. The 21-item Post-Traumatic Growth Inventory (PTGI) ${ }^{6}$ assessed the degree of personal growth (Cronbach's $a=0.956)$. Items are scored from 0 (I did not experience this change as a result of the COVID-19 lockdown) to 5 (I experienced this change to a very great degree as a result of the COVID-19 lockdown). Five subscale scores are produced: relating to others, new possibilities, personal strength, religion, and appreciation of life.

Personal resources/Predictors. The 6-item Brief Resilience Scale (BRS) ${ }^{17}$ assessed one's ability to bounce back or recover from stress (Cronbach's a=0.794). Items are scored from 1 (strongly disagree) to 5 (strongly agree). Items 2,4 , and 6 were reverse scored. The higher scores indicate higher resilience. The 28-item Coping Orientation to Problems Experienced Inventory (Brief COPE) ${ }^{18}$ assessed the frequency of emotion-focused (e.g., acceptance, emotional support, humor, positive reframing, and religion), problem-focused (e.g., active coping, instrumental support, and planning), and dysfunctional coping strategies (e.g., behavioral disengagement, denial, self-distraction, self-blame, substance use, and venting) (Cronbach's $a=0.842$ ). Items are scored from 1 (I have not been doing this at all) to 4 (I have been doing this a lot). For the scales that there was not a Greek version, a translation into Greek and back forth was completed, and results have been published. ${ }^{16}$

Instructions guided participants to respond to all items concerning their exposure to the COVID-19 traumatic event.

\section{Statistical analyses}

Descriptive statistics presented the sample sociodemographics. Cronbach's alpha assessed the internal consistency of the scales. Nearly all the study and outcome variables were not normally distributed (K-S: STS $=1.729$, $\mathrm{p}=0.005 ; \mathrm{PTG}=1,085, \mathrm{p}=0.190$ ) and Mann-Whitney $\mathrm{U}$ was used to examined differences between genders and levels of exposure to COVID-19. Correlation analy- ses were performed between a number of demographic variables, predictors and outcome variables with Spearman's rho. All significant correlations were entered in the regression analysis (stepwise method). All analyses with $p$-value $<0.05$ were considered significant and were performed with IBM SPSS 21.0.

\section{Results}

The majority of the HCWs reported that they have contact with infected patients (93.8\%) and/or diagnosed patients with COVID-19 (76\%) at work. A high percentage (79.3\%) reported at least moderate STS. HCWs had relatively low VPTG $(M=46.60, S D=24.61$, score range: $0-105)$ but high resilience $(M=21.65, S D=4.14$, score range: $8-30$ ). No differences were found neither for STS nor VPTG between levels of exposure (definitely being exposed or not exposed) to COVID-19 infected patients (STS: $U=6131.5, p=0.258$; VPTG: $U=5777, p=0.099$ ), and COVID diagnosed patients (STS: $U=11224, p=0.632$; VPTG: $U=11260, p=0.666)$. Resilience correlated with STS, age with VPTG, education and nearly all coping strategies with both STS and VPTG (table 1). Women had significantly higher scores than the men in the STS and VPTG $(U=32663, p<0.001$ and $U=32208, p<0.001$, respectively).

Table 1. Spearman's rho correlation coefficients between the outcome variables, resilience, and coping strategies among the healthcare workers $(\mathrm{N}=673)$.

\begin{tabular}{lcc}
\hline & STS & VPTG \\
\hline Age & -0.017 & $-0.087^{*}$ \\
Marital situation & 0.032 & -0.016 \\
Education & $-0.120^{* *}$ & $-0.137^{* *}$ \\
Self-distraction & $0.463^{* *}$ & $0.283^{* *}$ \\
Active coping & $0.190^{* *}$ & $0.298^{* *}$ \\
Denial & $0.550^{* *}$ & $0.207^{* *}$ \\
Substance use & $0.152^{* *}$ & $-0.107^{* *}$ \\
Use emotional support & $0.184^{* *}$ & $0.249^{* *}$ \\
Use instrumental support & $0.164^{* *}$ & $0.308^{* *}$ \\
Behavioral disengagement & $0.352^{* *}$ & -0.006 \\
Venting & $0.418^{* *}$ & $0.227^{* *}$ \\
Positive reframing & 0.003 & $0.330^{* *}$ \\
Planning & $0.138^{* *}$ & $0.208^{* *}$ \\
Humor & 0.068 & $0.093^{*}$ \\
Acceptance & -0.038 & $0.115^{* *}$ \\
Religion & $0.187^{* *}$ & $0.373^{* *}$ \\
Self-blame & $0.244^{* *}$ & $0.172^{* *}$ \\
Resilience & $-0.287^{* *}$ & 0.038 \\
\hline Note STS-Secondary Traumatic Str & $V P^{*}$ & \\
\hline
\end{tabular}

Note: STS=Secondary Traumatic Stress; VPTG= Vicarious PostTraumatic Growth, ${ }^{* *} \mathrm{p}<0.01,{ }^{*} \mathrm{p}<0.05$ 
Only HCWs that had contact with infected patients were included in the subsequent analyses (HCWs=633). Regression analyses (table 2) showed that STS was associated with one adaptive and three maladaptive coping strategies, explaining $40.6 \%$ of the variance. VPTG was associated with resilience, three adaptive and three maladaptive coping strategies, explaining $27.2 \%$ of the variance.

\section{Discussion}

The COVID-19 lockdown imposed a potentially more severe challenge than that of other mass crises, disasters, or infectious diseases that did not necessitate forced isolation. Despite the initial small numbers of confirmed cases and deaths in Greece, an upsurge occurred in August 2020. This reportedly "second wave" resulted in gradually imposing social distancing constraints. By the time of the last revision of this manuscript (January $11,2020)$ the exponential rise of confirmed cases and deaths in Greece (shorturl.at/rELYZ) leaded to a second lockdown. Finding the resources to withstand, respond effectively, recover, and move forward is of paramount importance. Therefore, this study examined the association of resilience and coping strategies with the positive and negative psychological effects of the COVID-19 first lockdown in a sample of HCWs in Greece.

Adequate evidence has shown that adaptive coping strategies are associated with lower rates of anxiety and PTSD, and maladaptive ones with increased risk. ${ }^{15,19}$ Avoidance (e.g., denial), a dysfunctional coping, has been associated with increased distress, especially during epidemics. ${ }^{19,20}$ In line with these findings, it was shown that dysfunctional coping strategies contributed to a higher risk of STS. The more the HCWs give up trying to deal with (behavioral disengagement), refuse to believe what is happening (denial) and turn to other activities not to think about it (self-distraction), the more STS. It has been suggested that when an outbreak is perceived as a severe life-threatening situation, then maladaptive coping strategies may be employed. ${ }^{12}$ Interestingly, a problem-focused strategy (i.e., planning) contributed to higher STS for HCWs. A possible explanation is that the COVID-19 outbreak was perceived as an uncontrollable disease, which caused feelings of inadequacy and helplessness. Given that no prevention or treatment means (e.g., vaccines) against COVID-19 had been developed during the first lockdown, being required to come up with a solution (e.g., planning) may go beyond the problem-solving abilities of the HCWs and cause them extremely high stress and frustration. ${ }^{20}$

In line with other studies that have shown that higher resilience was associated with lower anxiety levels ${ }^{21}$ and positive outcomes, 3,22 it was found that higher resilience was associated with lower STS levels (although the association was not retained in the regression analysis) and contributed to more VPTG (although the association was not initially significant in the bivariate correlations). Although one could expect that an acute stressor/trauma experience initially would decrease resilience and recovery would follow the wake

Table 2. Regression analyses ('stepwise') for predicting STS and VPTG for the healthcare workers who had contact with infected patients ( $\mathrm{N}=633)$, with coping strategies and resilience as predictors.

\begin{tabular}{|c|c|c|c|c|c|}
\hline Variables in the models & $\beta$ & $\mathrm{t}$ & $\mathrm{R}^{2}$ & Adjusted $\mathrm{R}^{2}$ & $\mathrm{R}^{2}$ Change \\
\hline \multicolumn{6}{|l|}{ I. STS (dependent) ${ }^{\mathrm{a}}$} \\
\hline Denial & 0.412 & $11.454^{* * *}$ & 0.317 & 0.316 & 0.317 \\
\hline Behavioral disengagement & 0.160 & $4.597^{* * *}$ & 0.400 & 0.397 & 0.019 \\
\hline Planning & 0.103 & $3.162^{* *}$ & 0.410 & 0.406 & 0.010 \\
\hline Religion & 0.225 & $6055^{* * *}$ & 0.132 & 0.131 & 0.132 \\
\hline Positive reframing & 0.188 & $4.861^{* * *}$ & 0.204 & 0.201 & 0.072 \\
\hline Self-distraction & 0.145 & $3.742^{* * *}$ & 0.236 & 0.232 & 0.032 \\
\hline Use instrumental support & 0.161 & $4.195^{* * *}$ & 0.256 & 0.251 & 0.020 \\
\hline Substance use & -0.116 & $-3.264^{* * *}$ & 0.267 & 0.260 & 0.011 \\
\hline
\end{tabular}

Note: STS=Secondary Traumatic Stress; VPTG=Vicarious Post-Traumatic Growth; ${ }^{* *} \mathrm{p}<0.01 ;{ }^{*} \mathrm{p}<0.05 ;{ }^{\mathrm{a}} \mathrm{F}=103.961<0.001 ;{ }^{\mathrm{b}} \mathrm{F}=33.068$, $p<0.001$ 
of adversity, ${ }^{20}$ this seems not to be the case with the HCWs. Abruptly being required to undertake the caring of the patients and breathlessly struggling with the infectious disease may have resulted in enhancement of their internal resources (e.g., rapid increase of their resilience), which in turn, may have resulted in more VPTG. However, this assumption needs to be examined. Long-term assessments could have given a clearer idea of this process.

As expected, PTG was associated with a number of adaptive coping strategies. The more they pray and meditate for stress relief (turn to religion), reconsider the situation (positive reframing), rely on their social networks for instrumental support, the more likely is VPTG to emerge. Indeed, religion and social support have been considered adaptive coping strategies in dealing with major life events. ${ }^{20,23}$

Substance use has been considered a risk factor for the deterioration of the mental health among trauma-exposed individuals to the pandemic. ${ }^{24}$ In line with these findings, substance use was expectedly associated with decreased VPTG. Another two maladaptive strategies (i.e., self-distraction and denial) that were associated with STS, also contributed to VPTG. Both distracting themselves to avoid thinking (self-distraction) and denying the situation (denial) seem to be beneficial. These findings are consistent with others, suggesting that avoidance and mainly emotional-focused strategies positively correlate with PTG. ${ }^{3}$ It seems that the so-called maladaptive coping strategies contribute to STS (behavioral disengagement, self-distraction, and denial) and PTG (self-distraction and denial). They may induce stress relief and thus, be equally effective in reducing negative outcomes and/or inducing posi-

\section{References}

1. Ploumpidis D. Living with covid-19. Psychiatriki 2020, 31:197, doi: 10.22365/jpsych.2020.313.197

2. Bride BE, Robinson MR, Yegidis B, Figley CR. Development and validation of the Secondary Traumatic Stress Scale. Res Soc Work Pract 2004, 14:27-35, doi: 10.1177/1049731503254106

3. Ogińska-Bulik N, Zadworna-Cieślak M. The role of resiliency and coping strategies in occurrence of positive changes in medical rescue workers. Intern Emerg Nursing 2018, 39:40-45, doi: 10.1016/j. ienj.2018.02.004

4. Kalaitzaki A, Tamiolaki A, Rovithis M. The healthcare professionals amidst COVID-19 pandemic: A perspective of resilience and post-traumatic growth. Asian J Psychiatr 2020, 52:102172, doi: 10.1016/j.ajp.2020. 102172

5. Batra K, Singh TP, Sharma M, Batra R, Schvaneveldt N. Investigating the psychological impact of COVID-19 among healthcare workers: tive ones. This may be especially true in traumatic situations of extreme threat, uncertainty, uncontrollability, feelings of hopelessness, and helplessness. Based on this assumption, it might be reasonable to assume that there are individually, subjective, responses depending on various factors, such as previous trauma exposure, perceived risk, and threat. Future studies should examine which strategies are successful responses to stress, no matter of their theoretical allocation to adaptive or maladaptive. Inter-specialty differences (physicians/ nurses vs. psychologists/social workers) also merit further research.

The limitations of this study should be acknowledged. The cross-sectional nature of this study does not allow one to say that these coping strategies were adaptive responses to lockdown or whether they were used before that. The convenience sampling and the underrepresentation of certain subgroups (e.g., men, older) does not permit the generalizability of the findings. The web-based self-administered questionnaire might have produced social desirability and selection bias (since primarily young and familiar with computers/cell phones were likely to respond). Not assessing symptoms of anxiety and depression, which have high comorbidity with PTSD, ${ }^{25}$ was also a serious limitation.

Considering the ongoing COVID-19 outbreak, safeguarding population subgroups with the tools to deal with would be of outmost importance. Enhancing resilience and encouraging successful coping strategies could be important targets by the stakeholders in developing efficient and effective prevention and intervention programs to safeguard those at risk and promote their posttraumatic growth.

A meta-analysis. Int J Environ Res Public Health 2020, 17:1-33, doi: 10.3390/ijerph17239096

6. Tedeschi RG, Calhoun LG. The Posttraumatic Growth Inventory: Measuring the Positive Legacy of Trauma. J Trauma Stress 1996, 9:455-471, doi: 10.1007/BF02103658

7. Mancini AD. Heterogeneous mental health consequences of COVID-19: Costs and benefits. Psychol Trauma 2020, 12:S15-S16, doi: $10.1037 /$ tra0000894

8. Huecker M, Shreffler J, Danzl D. COVID-19: Optimizing healthcare provider wellness and posttraumatic growth. Am J Emerg Med 2020, doi: 10.1016/j.ajem.2020.08.066

9. Shakespeare-Finch J, Bowen-Salter H, Cashin M, Badawi A, Wells R, Rosenbaum S et al. COVID-19: An Australian perspective. J Loss Trauma 2020, 25:662-672, doi: 10.1080/15325024.2020.1780748

10. Chen R, Sun C, Chen J, Jen H, Kang XL, Kao C et al. A large-scale survey on trauma, burnout, and posttraumatic growth among nurses during the COVID-19 pandemic. Int J Ment Health Nurs 2020, 30:102-116, doi: 10.1111/inm.12796 
11. Chew QH, Wei KC, Vasoo S, Sim K. Psychological and coping responses of health care workers toward emerging infectious disease outbreaks: A rapid review and practical implications for the COVID-19 pandemic. J Clin Psychiatry 2020, 81:20r13450, doi: 10.4088/ JCP.20r13450

12. Ye Z, Yang $X$, Zeng C, Wang Y, Shen Z, Li X et al. Resilience, social support, and coping as mediators between COVID-19-elated stressful experiences and acute stress disorder among college students in China. Appl Psychol Health Well Being 2020, 12:1074-1094, doi: 10.1111/aphw.12211.

13. Chew QH, Wei KC, Vasoo S, Chua HC, Sim K. Narrative synthesis of psychological and coping responses towards emerging infectious disease outbreaks in the general population: Practical considerations for the COVID-19 pandemic. Singapore Med J 2020, 61:350-356, doi: 10.11622/smedj.2020046

14. Cerami C, Santi G, Galandra C, Dodich A, Cappa S, Vecchi T et al. Covid-19 Outbreak in Italy: Are we ready for the psychosocial and economic crisis? Baseline findings from the Psycovid study. Front Psychiatry 2020, 11:556, doi: 10.3389/fpsyt.2020.00556

15. Savitsky B, Findling Y, Ereli A, Hendel T. Anxiety and coping strategies among nursing students during the covid-19 pandemic. Nurse Educ Pract 2020, 46:102809, doi: 10.1016/j.nepr.2020.102809

16. Kalaitzaki A. Posttraumatic symptoms, posttraumatic growth, and internal resources among the general population in Greece: A nation-wide survey amid COVID-19 first lockdown. Int J Psychol (accepted). Preprint, doi: 31234/osf.io/g29eb

17. Leontopoulou S. Resilience of Greek youth at an educational transition point: The role of locus of control and coping strategies as resources. Soc Ind Res 2006, 76: 95-126, doi: 10.1007/s11205-0054858-3
18. Kapsou M, Panayiotou G, Kokkinos CM, Demetriou AG. Dimensionality of Coping. J Health Psychol 2010, 15:215-229, doi: 10.2466/pr0.1988. 62.3 .763

19. Flesia L, Fietta V, Colicino E, Segatto B, Monaro M. Stable psychological traits predict perceived stress related to the COVID-19 outbreak. 2020, 9:3350, doi: 10.31234/osf.io/yb2h8

20. Babore A, Lombardi L, Viceconti ML, Pignataro S, Marino V, Crudele M et al. Psychological effects of the COVID-2019 pandemic: Perceived stress and coping strategies among healthcare professionals. Psychiatry Res 2020, 293:113366, doi: 10.1016/j.psychres.2020.113366

21. Savitsky B, Findling Y, Ereli A, Hendel T. Anxiety and coping strategies among nursing students during the covid-19 pandemic. Nurse Educ Pract 2020, 46:102809, doi: 10.1016/j.nepr.2020.102809

22. Breda AV. A critical review of resilience theory and its relevance for social work. Soc Work 2018, 54, doi: 10.15270/54-1-611

23. Imperatori C, Bersani FS, Massullo C, Carbone GA, Salvati A, Mazzi $G$ et al. Neurophysiological correlates of religious coping to stress: a preliminary eeg power spectra investigation. Neurosci Lett 2020, 728:34956, doi: 10.1016/j.neulet.2020.134956

24. Talarowska M, Chodkiewicz J, Biliński P, Nawrocka N, Miniszewska J. Mental health and the SARS-CoV-2 epidemic-Polish research study. Int J Environ Res Public Health 2020, 17:1-11, doi: 10.3390/ ijerph 17197015

25. Seto MC, Rodrigues NC, Ham E, Kirsh B, Hilton NZ. Post-traumatic stress disorder, depression, anxiety symptoms and help seeking in psychiatric staff: Trouble de stress post-traumatique, dépression, symptômes d'anxiété et recherche d'aide chez le personnel psychiatrique. Can J Psychiatry 2020, 65:577-583, doi: $10.1177 / 0706743720916356$ 


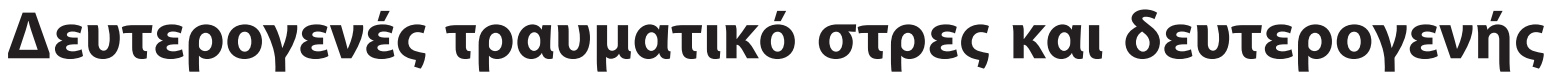

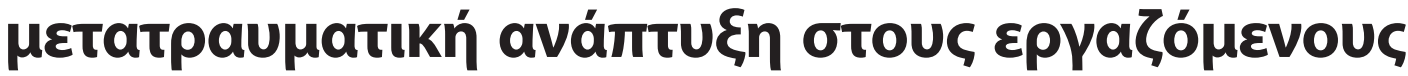

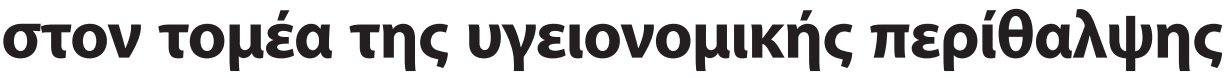

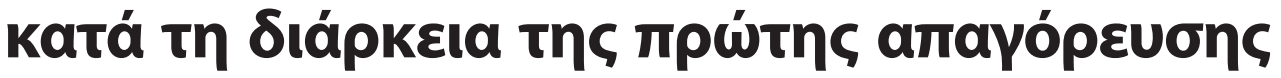

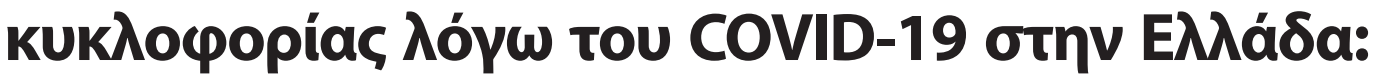

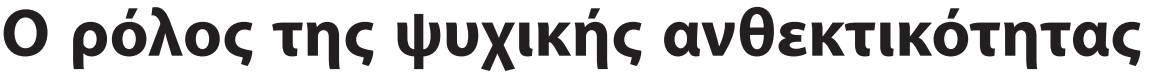

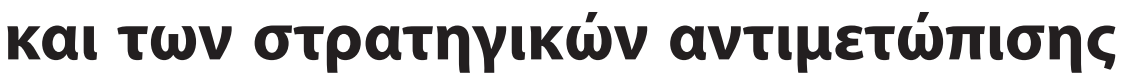

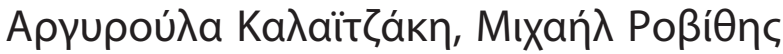

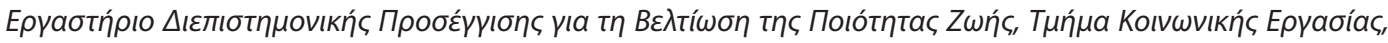

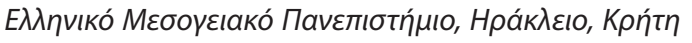

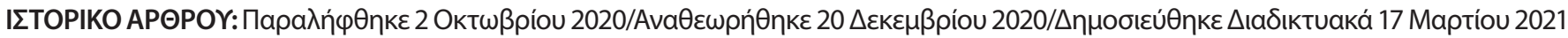

\section{ПЕРІАНЧН}

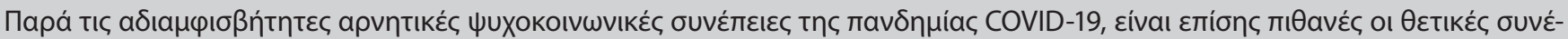

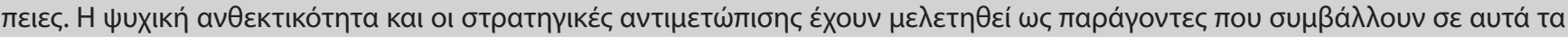

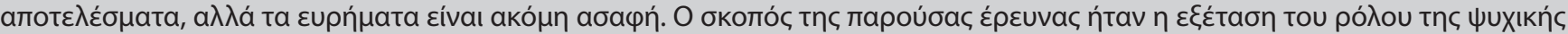

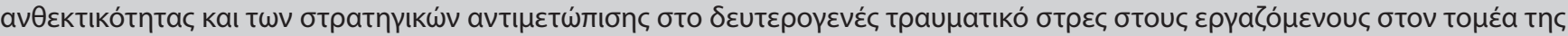

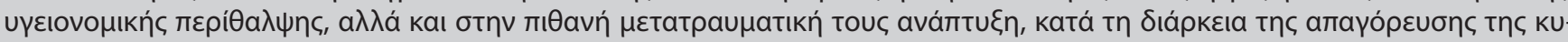

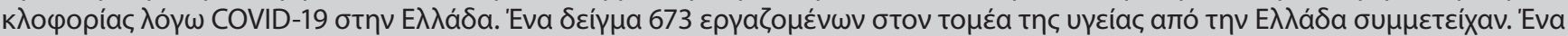

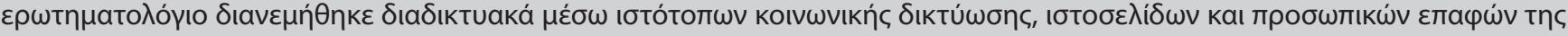

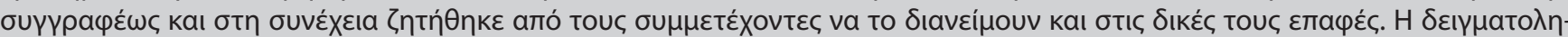

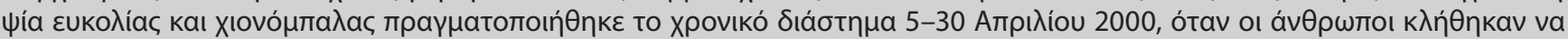

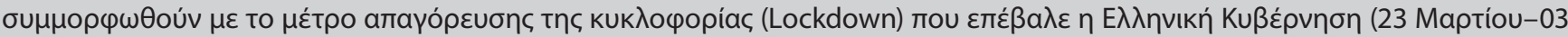

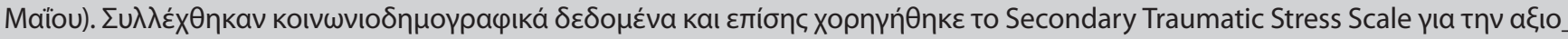

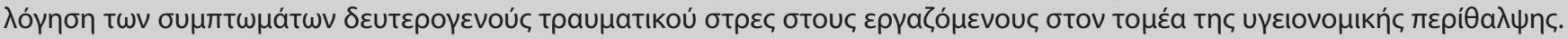
To Post-Traumatic Growth Inventory, to Brief Resilience Scale, kaı to Coping Orientation to Problems Experienced Inventory a६ı-

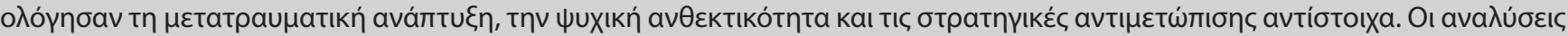

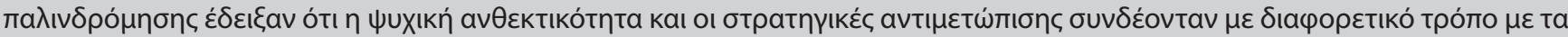

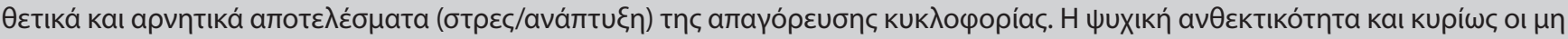

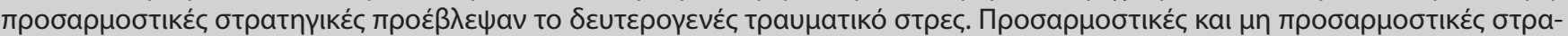

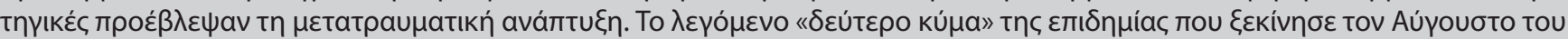

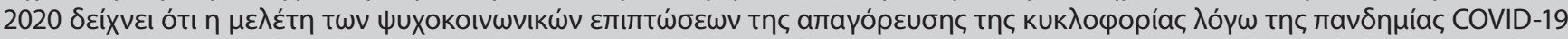

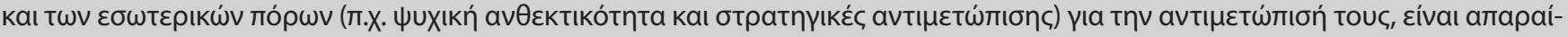

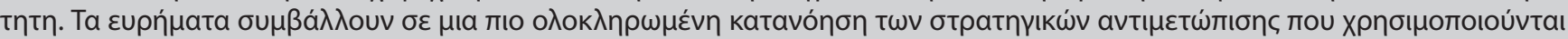

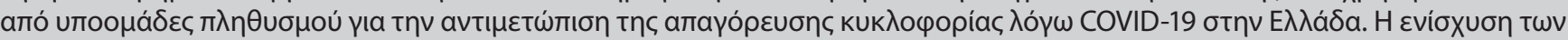

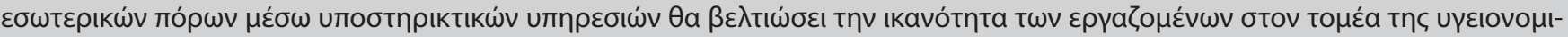

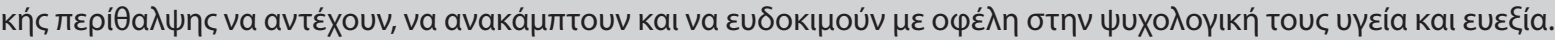

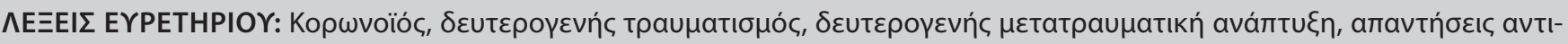

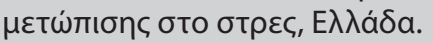

